\title{
Implementación de un robot móvil seguidor de línea y detector de obstáculos con comunicación Bluetooth
}

Fecha de recepción: 2 de septiembre de 2016

Fecha de aprobación: 9 de diciembre de 2016 Pp. 99-118
Camilo Mejía Moncayol Universidad EAN cmejiam@ean.edu.co

Luis Armando Cobo Campo 2

Universidad EAN

lacobo@universidadean.edu.co

Hebert Alberto Calderón 3

Universidad EAN

hcaldero2890@universidadean.edu.co

DOI:https://doi.org/10.21158/23823399.v4.n2.2016.1639

Cómo citar este artículo/ To reference this article/Comment citer cet article/ Para citar este artigo: Mejía Moncayo, C.; Cobo, L. y Calderón, H. A. (2016). Implementación de un robot móvil seguidor de línea y detector de obstáculos con comunicación Bluetooth. Revista Ontare, 4(2), 99-118. DOI: https:// doi.org/10.21158/23823399.v4.n2.2016.1639

\section{Resumen}

Este artículo presenta el proceso de desarrollo de un prototipo de robot móvil, que cuenta con comunicación Bluetooth y puede operar en dos modos: el primero como seguidor de línea, en el que sigue una trayectoria definida por una línea negra dibujada sobre una superficie blanca. El segundo modo de operación es como detector de obstáculos, en el que se mueve hacia adelante tratando de evitar los obstáculos que se le presentan en el camino. El prototipo final que se obtuvo trabaja correctamente en los dos modos, y sirve de plataforma de desarrollo de vehículos guiados automáticamente.

\section{Palabras clave}

Robot móvil, seguidor de línea, detector de obstáculos, Bluetooth, Arduino.

\footnotetext{
${ }^{1}$ Magister en Ingeniería mecánica de la Universidad Nacional de Colombia. Ingeniero mecánico de la Universidad Nacional de Colombia. ORCID: http://orcid.org/0000-0002-3661-2231

2 Ph.D. en Ingeniería, Universidad de los Andes y en Génie Informatique, École Polytechnique de Montréal. Magister en Ingeniería de Sistemas y Computación, Universidad de los Andes. ORCID: http://orcid.org/0000-0003-3034-101X .

3 Estudiante del programa de Ingeniería de producción de la Universidad EAN.
} 


\title{
Implementation of a mobile robot with Bluetooth communication that can operate as a line follower and an obstacle detector
}

\begin{abstract}
This article presents the development process of a mobile robot prototype, with Bluetooth communication that can operate in two modes: the first one, as a line follower, moving along a path that has been defined by a black line drawn on a white surface. The second mode of operation is as an obstacle detector, in which it moves forward trying to avoid the obstacles that appear on the way. The final prototype that was obtained works correctly in both modes, and serves as a platform for the development of automatically guided vehicles.
\end{abstract}

\section{Keywords}

Mobile robot, line follower, obstacle detector, Bluetooth, Arduino.

\section{Implémentation d'un robot mobile détecteur d'obstacles et de démarcations au sol interagissant par communication Bluetooth}

\section{Résumé}

Cet article présente le processus de développement d'un prototype de robot mobile disposant de communication Bluetooth et pouvant agir de deux manières: comme suiveur de démarcation au sol utilisant une trajectoire définie par une ligne noire dessinée sur une surface blanche; et comme détecteur d'obstacles, avançant en essayant d'éviter les obstacles se présentant à lui. Le prototype final fonctionne correctement dans les deux modalités et sert de plate-forme de développement de véhicules à guidage automatique.

\section{Mots clefs}

Robot mobile, suiveur de démarcations, détecteur d'obstacles, Bluetooth, Arduino. 


\section{Implementação de um robô móvel com comunicação Blue- tooth seguidor de linha e detector de obstáculos}

\section{Resumo}

Este artigo apresenta o processo de desenvolvimento de um protótipo de robô móvel, que conta com comunicação Bluetooth e pode operar de dois modos: o primeiro, como seguidor de linha, no qual segue uma trajetória definida por uma linha negra desenhada sobre uma superficie branca; o segundo modo de operação é como detector de obstáculos, no qual se move para adiante tratando de evitar os obstáculos que se apresentam no caminho. O protótipo final que se obteve trabalha corretamente nos dois modos, e serve de plataforma de desenvolvimento de veículos guiados automaticamente.

\section{Palavras-chave}

Robô móvel, seguidor de linha, detector de obstáculos, Bluetooth, Arduino. 


\title{
1. Introducción
}

\begin{abstract}
lo largo de la historia el ser humano ha tenido la necesidad de Aconstruir máquinas y herramientas para realizar el trabajo más pesado, rutinario, peligroso y preciso. Esto dio origen al desarrollo de los robots para ejecutar estas y otras muchas tareas, buscan evitar la intervención humana. Una de ellas es el desarrollo de sistemas móviles que sigan un recorrido de forma autónoma, guiados por sensores o rutas que han sido cargadas o enviadas mediante un sistema de comunicación. En este contexto se presenta este trabajo, donde se exponen los resultados finales de un proyecto del semillero de robótica de la Universidad EAN, en el que se desarrolló un robot móvil seguidor de línea y detector de obstáculos con comunicación Bluetooth, empleando las plataformas Arduino y Android.
\end{abstract}

El desarrollo de robots móviles responde a la necesidad de incrementar la autonomía para limitar todo lo posible la intervención humana. La autonomía de un robot móvil se basa en el sistema de navegación automática; en estos sistemas se incluyen tareas de planificación, percepción y control (Baturone, 2005), como las que ejecuta un seguidor de línea, que cuenta con sensores infrarrojos que detectan la línea, y mediante un dispositivo digital de control (en este caso un Arduino) realizan una acción de control establecida en un programa que dirige al robot en una secuencia de órdenes para que este mantenga la trayectoria demarcada. Algunas de las aplicaciones de este tipo de robots las encontramos en instalaciones como un hospital (Jain, Sharma, \& Chauhan, 2014) y en aplicaciones de vehículos de altas velocidades (Khafri \& Jahanian, 2012). Un punto interesante ha sido la utilización de este tipo de robots con fines didácticos y pedagógicos tanto en escuelas como en universidades. 
El artículo de Pakdaman, Sanaatiyan, \& Ghahroudi (2010) presenta las experiencias en el diseño y pruebas del robot seguidor de línea llamado TABAR. Entre las conclusiones que podemos observar en este trabajo están las experiencias positivas que implicó para los estudiantes el desarrollo de este proyecto y la satisfacción obtenida por estos en la realización del robot. Otro importante experimento es planteado por los autores Bajestani \& Vosoughinia (2010). En tal trabajo, que se trata de un estudio preliminar en la construcción de robots seguidores de línea, se demuestra la gran utilidad que tienen estos robots en la formación de estudiantes, al combinar en un solo experimento el desarrollo de hardware con la construcción de aplicativos útiles para ellos. Otros trabajos que demuestran la utilidad de los robots seguidores de línea en la educación los podemos ver en Su, Lee, Huang, Chuang, \& Lin (2010) y Cruz-Álvarez, Hidalgo-Peña y Acosta-Mesa (2012). De igual forma, la detección de obstáculos con sensores ultrasónicos permite ubicar objetos en una ruta empleando ondas sonoras, con lo cual un robot puede definir en tiempo real una ruta que evite una colisión; esta capacidad es muy importante en la vida real y es necesario resaltar que esta tecnología es ampliamente usada en vehículos comerciales para facilitar el parqueo y otras aplicaciones, como la movilidad para invidentes (Egaña, Ceres, Seco, \& Pons, 2004).

Por último, la comunicación es un proceso indispensable en cualquier aplicación moderna de robótica, ya que a través de esta es posible monitorear y controlar dispositivos en tiempo real. Una de las tecnologías más comunes es el Bluetooth, que permite generar una red inalámbrica entre dispositivos móviles (Amin, bt Fisal, \& Bakar, n.d.), lo cual facilita la interacción entre Smartphones y robots a través de aplicaciones como Bluetooth terminal, empleada en este proyecto.

En este trabajo se presenta el desarrollo de un prototipo de un robot seguidor de línea que fue resultado de un periodo de investigación y consulta, y que tiene como características adicionales la detección de objetos y el control remoto por medio de comunicación Bluetooth 
con un teléfono celular. Esto con el fin de dar una visión proyectada al área industrial y no solamente como un prototipo para muestra de ensayo y error.

El artículo a continuación está organizado de la siguiente manera: en la sección 2 se presentan los detalles de implementación del prototipo de robot, indicando los recursos utilizados para su construcción, así como la metodología empleada para el desarrollo del robot; la sección 3 presenta las diversas pruebas realizadas al prototipo y se especifican los resultados obtenidos; la sección 4 relaciona las conclusiones al trabajo realizado.

\section{Implementación del proyecto}

Dara el desarrollo de este trabajo inicialmente se definieron los objetivos, luego se realizó la planeación de las diferentes fases del proyecto, las cuales se enumeran a continuación y posteriormente se describen de una forma más detallada. El proceso implementado se soportó en la investigación bibliográfica y experimentación para lograr el prototipo final.

Las actividades que se definieron para el desarrollo del proyecto fueron las siguientes:

- Planeación.

- Diseño y construcción mecánica del prototipo.

- Diseño y estructuración del sistema electrónico.

- Desarrollo del código o módulos de programación.

- Ensamble de los diferentes sistemas y componentes del robot.

- Pruebas y resultados.

- Prototipo final. 


\subsection{Diseño y planeación del prototipo}

En esta etapa se definieron las características físicas del prototipo, la tipología y las posiciones donde iban a serubicados los sensores, actuadores y la lógica general de este. Como parte inicial para el desarrollo de esta etapa se estableció el tipo de robot móvil que se iba a construir. Para este caso se decidió por un robot móvil seguidor de línea, con detección de obstáculos y comunicación Bluetooth. Este consiste básicamente de dos motores con ruedas como tracción y una rueda loca de guía, una base donde va todo el sistema electrónico, los sensores, actuadores y el módulo de comunicación.

\subsection{Construcción del prototipo}

Una vez determinados los elementos que se iban a utilizar, se inició la construcción del prototipo, es decir específicamente, el ensamble de la estructura mecánica, para lo cual se empleó un Magician Chassis DG007, que consiste en un conjunto de piezas que al ser armadas conforman todo el sistema mecánico del carro, ya que trae dos bases, dos ruedas con motores, separadores, tornillos, tuercas y una rueda loca para su libre desplazamiento (Figura 1). 
Figura 1. Ensamble mecánico final y muestra del montaje con la protoboard y el circuito.
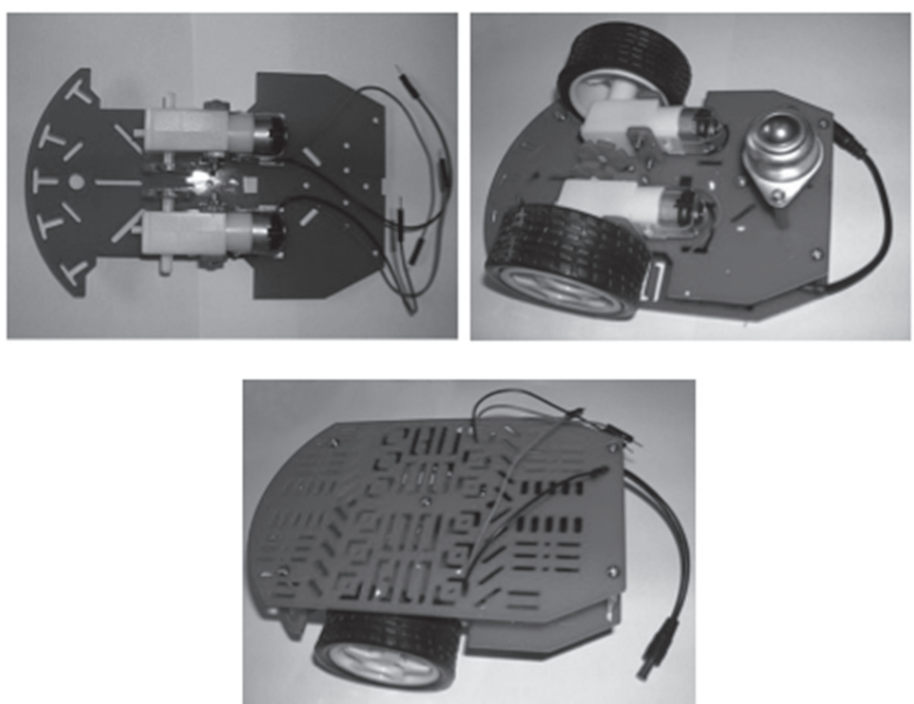

Fuente. Elaboración propia.

\subsection{Diseño y estructuración del sistema electrónico}

Para el desarrollo de esta etapa se realizó el diseño y simulación del circuito electrónico empleando Fritzing y Proteus (Monk, 2015), tal como se puede apreciar en las figuras 2 y 3 . Mediante las simulaciones fue posible establecer la potencia consumida por los motores, el amplificador operacional, los sensores, la placa Arduino, el módulo Bluetooth y los demás componentes. A continuación, se realiza una descripción general de los principales componentes del sistema:

Fuente de alimentación: el circuito inicia con la fuente de alimentación (batería 9V), la que energiza todo el sistema; luego de esto pasa por un interruptor de codillo de dos posiciones, que funciona como encendido/apagado de todo el sistema electrónico, posteriormente la fase positiva pasa a un diodo $1 \mathrm{~N} 4004$ rectificador y luego se ramifica en dos partes: una se dirige al pin 8 (Vs) del driver L293D para 
energizar los motores, y la segunda va al regulador 7805 para reducir los $9 \mathrm{~V}$ de entrada a $5 \mathrm{~V}$. Una vez definidas las líneas de $5 \mathrm{~V}$ y tierra, culmina la etapa de alimentación y potencia.

Figura 2. Circuito electrónico con componentes físicos elaborado en Fritzing.

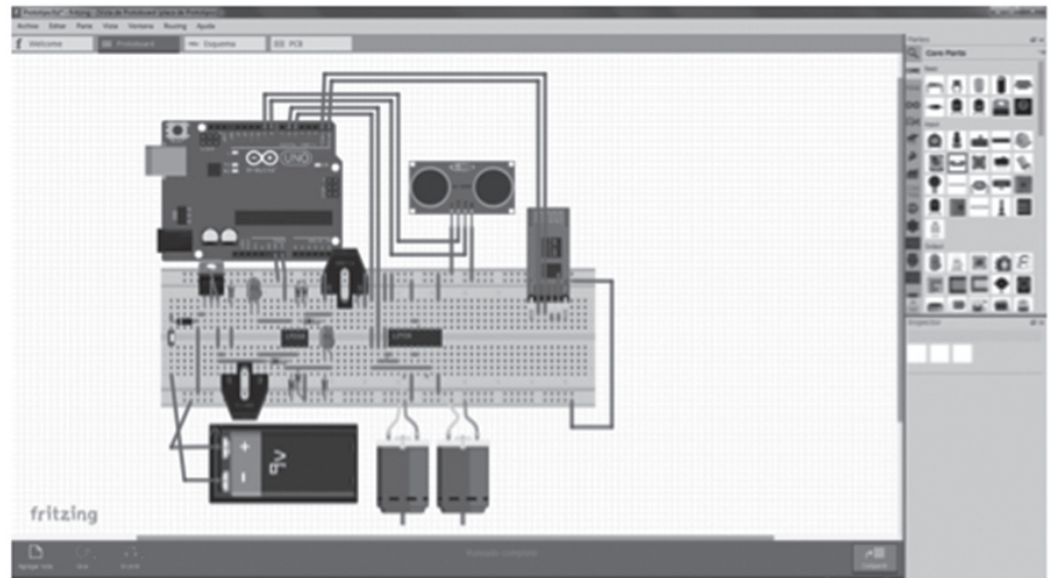

Fuente. Elaboración propia.

Figura 3. Circuito electrónico con simbología para interpretación en Fritzing.

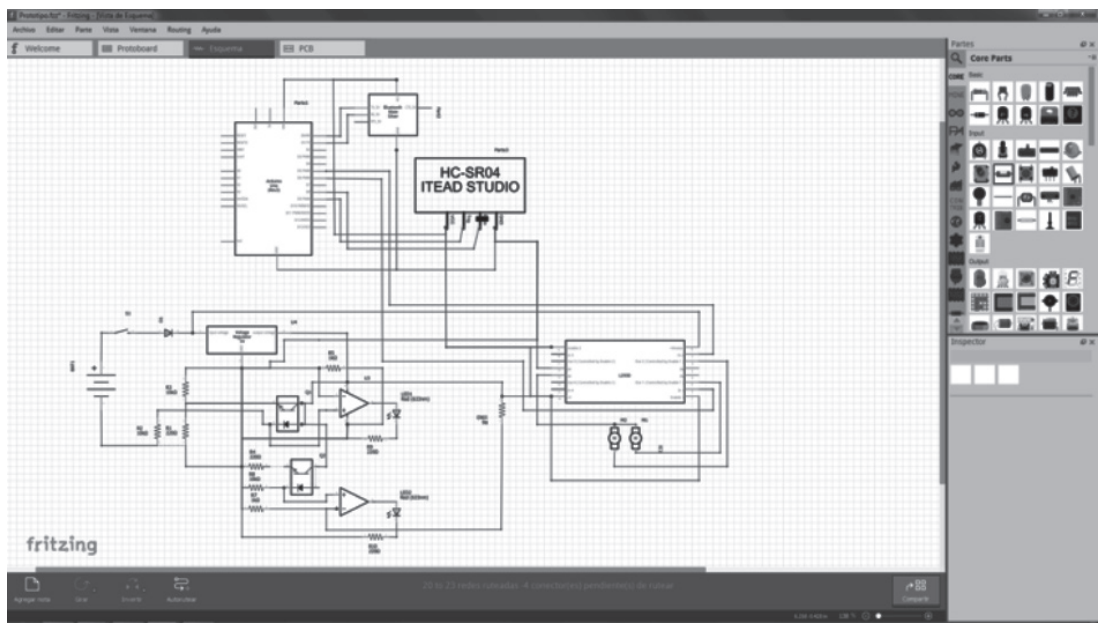

Fuente. Elaboración propia. 
Sensores infrarrojos: la señal de salida de los sensores QRD-1114 se obtuvo y gestionó utilizando un amplificador operacional (AO) de referencia LM 358, donde fue configurado como comparador (figura 4), es decir, que se ingresan dos voltajes: uno en cada una de las entradas del AO y se comparan. Cuando V1 es mayor que V2, la salida en Vout es $\mathrm{Vs}^{+}$, pero si V1 es menor que V2 la salida en Vout será Vs-. Por lo tanto, se dejó un voltaje V1 como la salida que emite el sensor por la terminal del cátodo (voltaje variable), y el voltaje V2 quedó como el resultante que proviene de estar en medio de dos resistencias: una de $10 \mathrm{~K} \Omega$ y otra de $1 \mathrm{~K} \Omega$ (voltaje de referencia), de esta manera se garantizará que su salida lógica sea de un $0 \mathrm{~V}$ o $5 \mathrm{~V}$ y pueda así ingresar sin problemas al Arduino. Es importante aclarar que el sensor QRD-1114 es un fototransistor, donde una parte emite y la otra la recibe el reflejo de la luz emitida, que posee cuatro terminales: ánodo, cátodo, colector y emisor.

Figura 4. Amplificador operacional comparador.

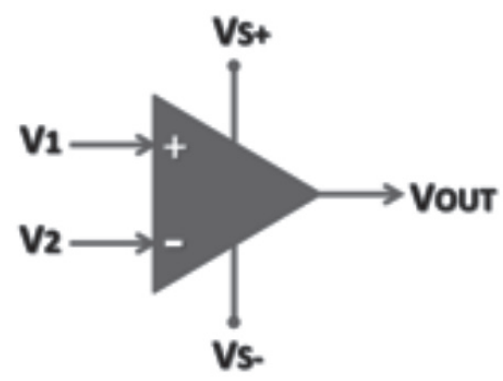

Fuente. Elaboración propia.

Sensores ultrasónicos: posteriormente se realizó la conexión de los sensores ultrasónicos HC-SR04 a la placa Arduino, los cuales permiten detectar obstáculos mediante un emisor (altavoz) que envía una señal sonora, que luego el receptor (micrófono) recibe; el tiempo que transcurre entre la señal emitida y la recibida es proporcional a la distancia del objeto al sensor (Figura 5). 
Módulo Bluetooth HC-06: este módulo se conecta a los pines RX y Tx del Arduino, para permitir la comunicación inalámbrica.

Figura 5. Esquemático de los sensores infrarrojos con amplificador operacional.

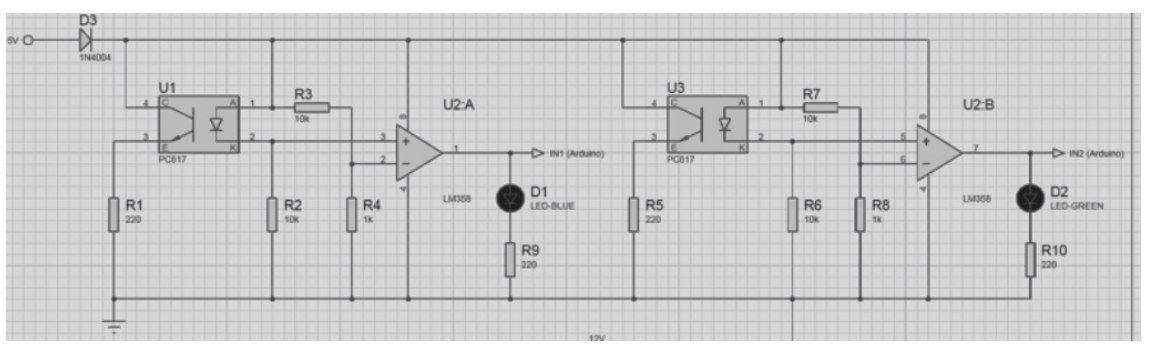

Fuente. Elaboración propia.

\subsection{Programación de la placa Arduino}

Dadas las tres tareas que debía cumplir el robot: seguir una línea, detectar obstáculos y establecer comunicación Bluetooth, se desarrolló la programación del robot en Arduino IDE, con una estructura modular, con funciones que trabajan dependiendo del modo seleccionado por el usuario. Bajo esta lógica se estructuró el programa principal que se presenta en la figura 6 en diagrama de flujo, en términos generales este inicia con el proceso de comunicación con el módulo Bluetooth a través del cual se le puede definir al robot si debe trabajar en modo seguidor de línea o detector de obstáculos. Esta verificación se realiza todo el tiempo y permite al usuario cambiar de modo de operación cuando lo desee, para lo que solo debe comunicarse con el robot mediante la aplicación Bluetooth terminal y enviar la letra que identifica el modo de operación. A continuación, se exponen los procesos que realiza el programa principal (Figura 6). 
Figura 6. Diagrama programa principal

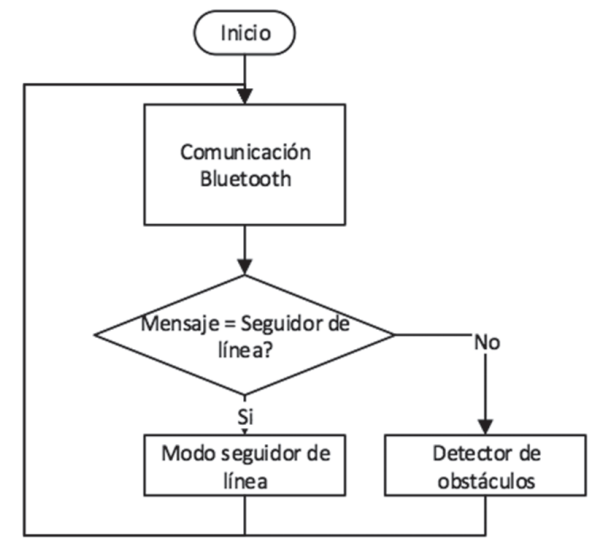

Fuente. Elaboración propia.

El proceso de comunicación Bluetooth verifica si fue enviado un mensaje, si la respuesta es afirmativa realiza la lectura del mensaje y lo transfiere al programa principal para que se seleccione el modo de trabajo del robot (Figura 7).

Figura 7. Comunicación Bluetooth.

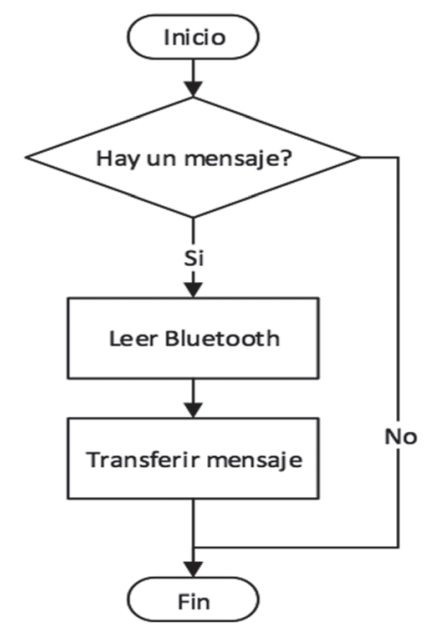

Fuente. Elaboración propia. 
Si el modo seleccionado es seguidor de línea, el robot verifica los estados de los dos sensores infrarrojos (derecho e izquierdo), y a partir de las posibles combinaciones de estados de estos realiza sus movimientos, como se presenta en la figura 8 . La primera alternativa corresponde a la situación en la que los dos sensores detecten la línea negra, en este caso el robot se mueve hacia adelante, si el robot solo detecta la línea con uno de los sensores, corrige la dirección girando en el sentido del sensor que está detectando la línea para alinearse y continuar siguiendo la línea, en caso de que el sensor no detecte la línea con ninguno de los sensores, el robot gira hasta que localice la línea.

Figura 8. Modo seguidor de línea.

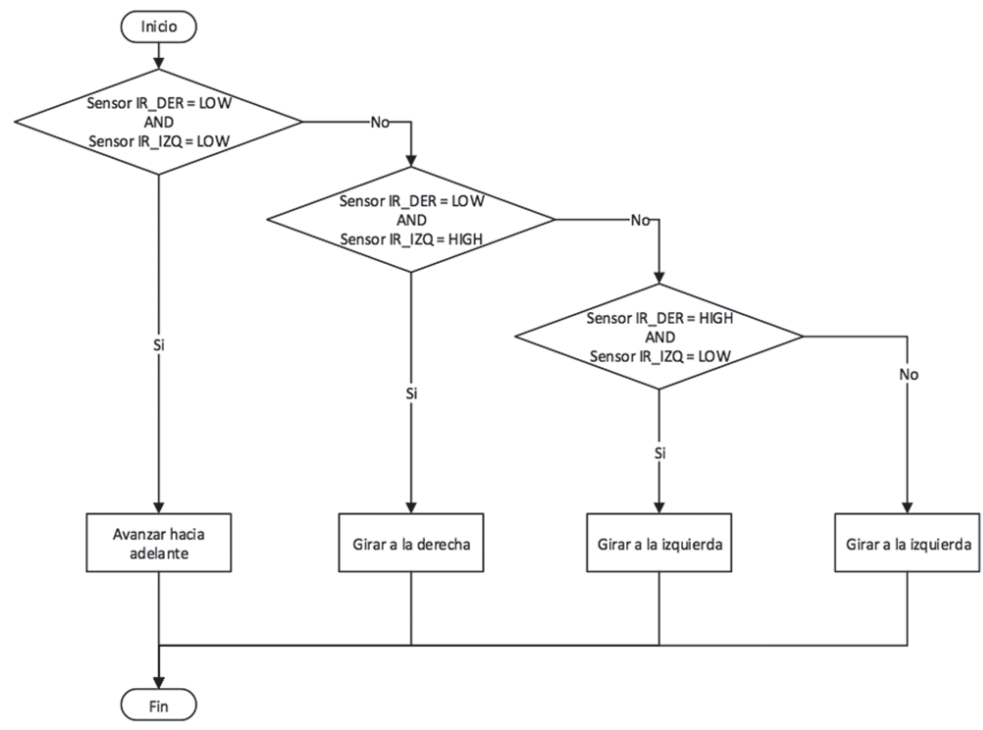

Fuente. Elaboración propia.

Si el robot está en el modo de detector de obstáculos, avanza hacia adelante hasta que localiza uno de los sensores ubicados a la derecha o a la izquierda, la rutina que realiza se fundamenta en girar en sentido opuesto al obstáculo, hasta lograr una trayectoria libre de estos, el proceso realizado se presenta en la figura 9. 
Figura 9. Modo detector de obstáculos.

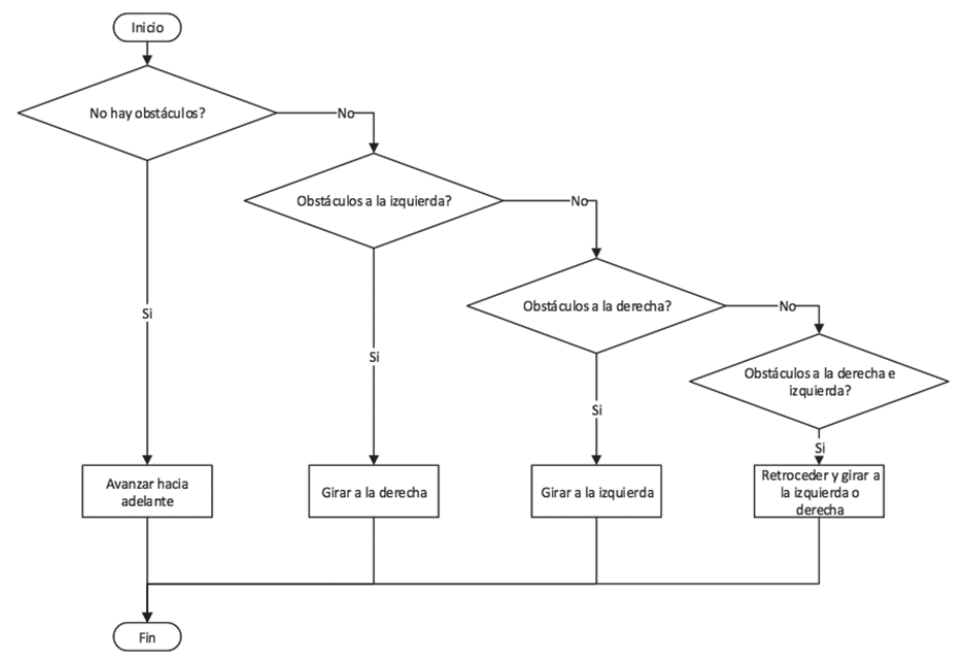

Fuente. Elaboración propia.

\subsection{Ensamblaje final de los diferentes sistemas $y$ componentes del robot}

En esta etapa se monta cada uno de los componentes al chasis para obtener el ensamble final de robot. El orden del ensamble fue el siguiente:

- Ensamble de los componentes electrónicos, lo cual implicó realizar las conexiones de los sensores y actuadores al Arduino.

- Montaje en el chasis de los circuitos y Arduino al chasis del robot.

Por último, se realizaron los últimos ajustes y puesta a punto de todos los componentes para proceder con las pruebas del prototipo construido (Figura 10). 
Figura 10. Ensamblaje final de los sistemas y componentes del robot.

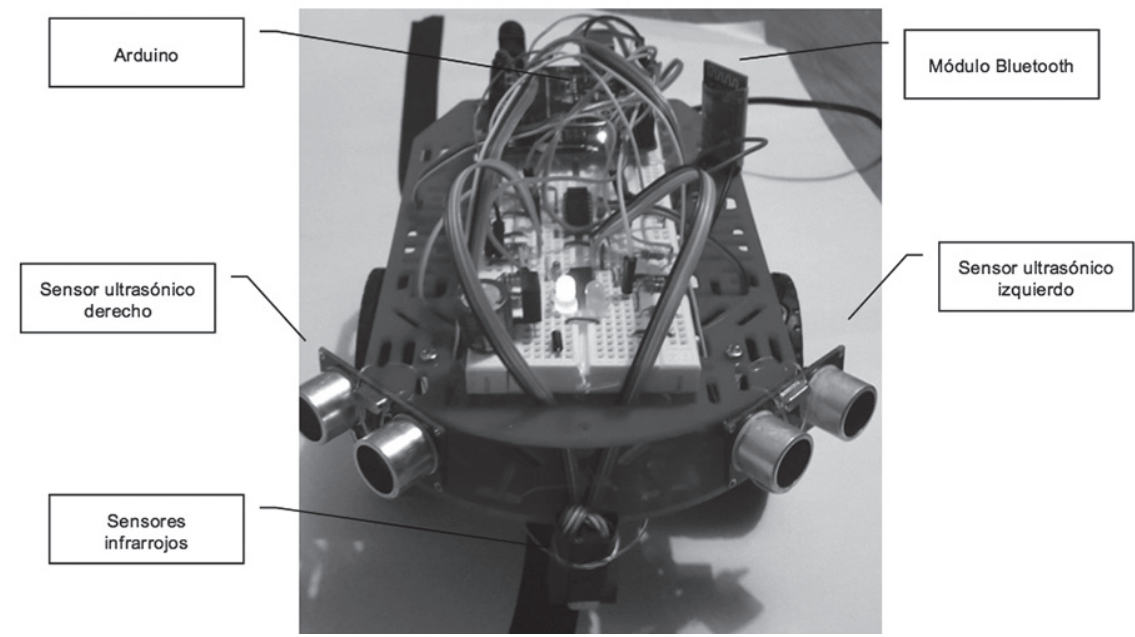

Fuente. Elaboración propia.

\section{Pruebas y resultados}

Tna vez terminado el ensamble de todos los sistemas se procedió a realizar las pruebas de funcionamiento. Estas incluyeron la verificación individual de las funciones de comunicación Bluetooth, modo seguidor de línea y detector de obstáculos, para finalizar con una prueba simultánea de todas las funciones.

Primero se realizó la comunicación vía Bluetooth entre el celular y el Arduino por medio de la aplicación móvil Bluetooth Terminal, luego se envió la letra $\mathrm{H}$, que activa el modo seguidor de línea, donde se verificó que el carro siguiera una ruta establecida sin que llegara a presentarse una interrupción del movimiento (figura 11). Cabe tener en cuenta que la distancia de lectura realizada por los sensores infrarrojos es variable, por lo que fue necesario efectuar su calibración para evitar problemas durante la puesta en marcha. 
Figura 11. Pruebas de funcionamiento del modo seguidor de línea.
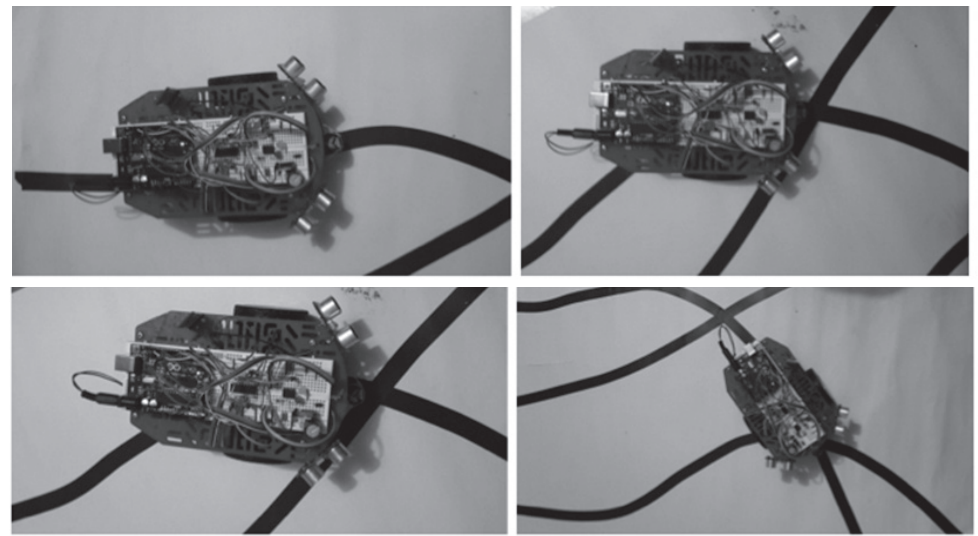

Fuente. Elaboración propia.

Después de haber verificado el funcionamiento del robot móvil como seguidor de línea, se continuó con la revisión y puesta en marcha del modo detector de obstáculos, el cual se activa al enviar al robot la letra G; como evidencia de esto se observó que el robot cambiaba de ruta cada vez que encontraba un objeto durante su recorrido, siguiendo las instrucciones definidas en el programa (Figura 12).

Figura 12. Pruebas de funcionamiento del modo detector de obstáculos.
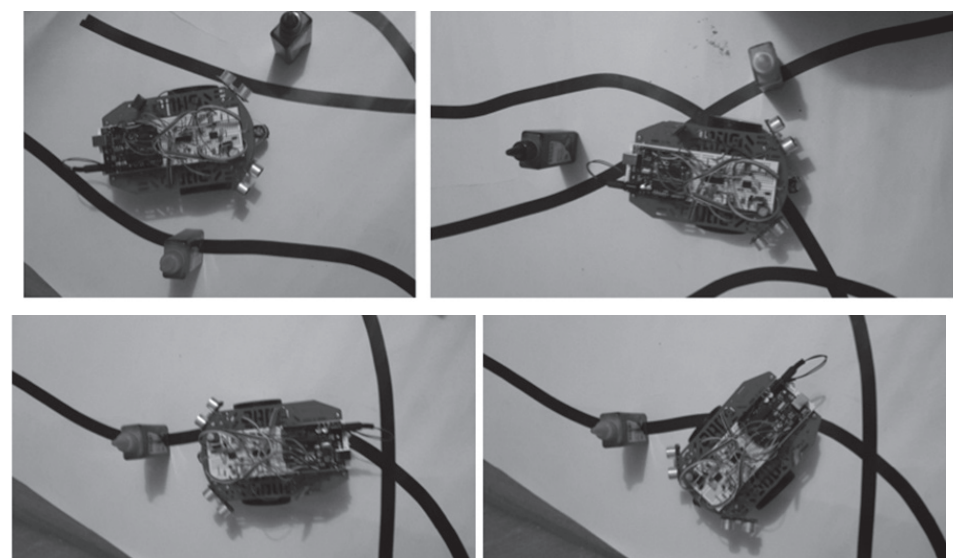

Fuente. Elaboración propia. 
Camilo Mejía Moncayo

Luis Armando Cobo Campo

Hebert Alberto Calderón

\subsection{Prototipo final}

El prototipo obtenido después de finalizar las pruebas y la puesta a punto de los sistemas se presenta en la figura 13.

Figura 13. Prototipo final.

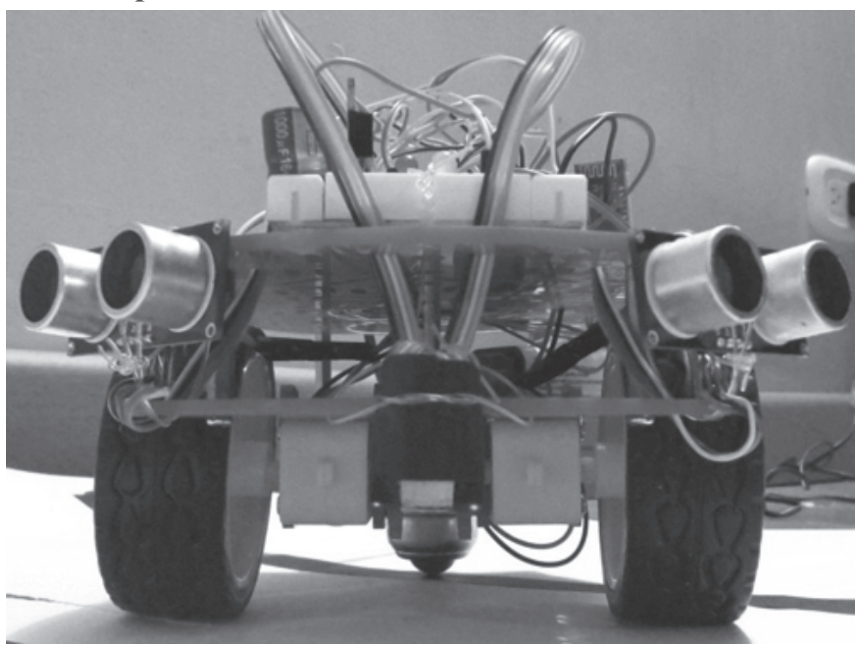

Fuente. Elaboración propia. 


\section{Conclusiones}

1 resultado principal del prototipo desarrollado en este trabajo fue Clograr una herramienta que funciona de forma satisfactoria y sirve para la introducción de los estudiantes en la implementación de vehículos guiados automáticamente.

Otro de los aportes de este trabajo es establecer una arquitectura a partir de la cual se pueden implementar sistemas de distribución de tipo didáctico, que integrados con manipuladores y sistemas de almacenamiento permiten lograr el desarrollo de un sistema logístico automático.

Se identificó que uno de los principales inconvenientes del diseño actual del robot móvil tiene que ver con el gasto excesivo de la batería al utilizarse de forma permanente el módulo Bluetooth conectado a la placa Arduino. Este módulo tiene un consumo superior de energía al esperado. En futuras versiones del proyecto realizaremos investigaciones sobre las maneras eficientes de acoplar estos módulos de comunicación inalámbrica al robot, que permitan un consumo adecuado de la batería utilizando esquemas; por ejemplo, de dormir el dispositivo y despertarlo solo cuando sea necesario.

Finalmente, la metodología implementada demostró ser exitosa y los resultados obtenidos de este prototipo sirven como semilla para nuevos proyectos a los que se les pueden integrar funciones adicionales a las aplicadas en este prototipo. 


\section{Referencias}

Che Fai, Y.; Amin, S. H.; Fisal, N. \& Bakar, J. A. (2002). Bluetooth enabled mobile robot in 2002. IEEE International Conference on Industrial Technology, IEEE, 2, pp. 903-908.. http://doi.org/10.1109/ ICIT.2002.1189288

Bajestani, S., \& Vosoughinia, A. (2010). Technical report of building a line follower robot. International Conference On Electronics and Information Engineering (ICEIE), Kyoto: IEEE.

Baturone, A. O. (2005). Robótica: manipuladores y robots móviles. Marcombo. Recuperado de https://books.google.com/ books? id=TtMfuy $6 \mathrm{FNCcC} \&$ pgis $=1$

Cruz-Álvarez, V. R., Hidalgo-Peña, E., \& Acosta-Mesa, H.-G. (2012). A line follower robot implementation using Lego's Mindstorms Kit and Q-Learning. Acta Universitaria, 22, 113-118. Recuperado de http:// www.redalyc.org/resumen.oa? $\mathrm{id}=41623190016$

Egaña, A., Ceres, R., Seco, F., \& Pons, J. L. (2004). Detección ultrasónica de obstáculos por medida diferencial para la movilidad de invidentes. XXV Jornadas de Automática Ciudad Real, 7.

Jain, T., Sharma, R., \& Chauhan, S. (2014, December 1). Applications of Line Follower Robot in Medical Field. International Journal of Research. Recuperado de http://internationaljournalofresearch.org/ index.php/ijr/article/view/1133

Khafri, Y. Z., \& Jahanian, A. (2012). Improved Line Tracking System for Autonomous Navigation of High-Speed Vehicle, IAES, 1(3), 163 174. http://dx.doi.org/10.11591/ijra.v1i3.766 
Monk, Simon (2015). Fritzing for Inventors: Take Your Electronics Project from Prototype to Product. McGraw-Hill Education

Pakdaman, M., Sanaatiyan, M. M., \& Ghahroudi, M. R. (2010). A line follower robot from design to implementation: Technical issues and problems. The 2nd International Conference on Computer and Automation Engineering. Singapore: IEEE. (ICCAE), 5-9.

Su, J. H., Lee, C. S., Huang, H. H., Chuang, S. H., \& Lin, C. Y. (2010). An intelligent line-following robot project for introductory robot courses. World Transactions on Engineering and Technology Education, 8(4), 455-461. 